\title{
Trade-off modeling of superconducting levitation machines: theory and experiment
}

\author{
A. Badía-Majós, A. Aliaga, J. Letosa, M. Mora and J. Peña
}

\begin{abstract}
Based on the critical state model for the superconducting components, we develop a set of theoretical tools that allow to extract relevant engineering parameters of a superconducting levitation machine. We provide a number of analytical and numerical expressions for the evaluation of the electromagnetic quantities, energies and forces in $2 \mathrm{D}$ problems. This assumption includes: (i) rotational symmetric systems as those in bearings and motors, and also the case of (ii) translational symmetry as in long transportation lines. The theory, that trades off simplicity and predictive power builds on the vector potential/current density formulation of the Maxwell equations $(\mathrm{A}, \mathrm{J})$ and is validated by comparison against experimental tension-compression data in our universal test machine. As shown, very simple computer coding is required to implement the method.
\end{abstract}

Index Terms-Critical State Model, Magnetic Levitation Force, Superconducting Modeling.

\section{INTRODUCTION}

Magnetically levitated devices based on permanent magnets and superconductors have received increasing attention over the last years and a number of possible configurations have been described [1]. The intrinsic properties of the high- $\mathrm{T}_{c}$ materials, that are already available at the commercial level with physical parameters suitable for large scale applications, give way to unique levitation characteristics. In particular, selfstabilization against arbitrary perturbations, provided by the flux-pinning property is to be mentioned. At the engineering level, the evaluation of performances involves to consider a number of design parameters such as the maximum forces and stiffness coefficients attainable. Concerning the numerical simulations, the incorporation of the superconducting material law in the electromagnetic equations, with a reasonable predictive capacity has been the motivation of a number of works. A very complete and comprehensive compilation of the different advances in this field may be found in [2]. In brief, the authors of that paper classify the hierarchy of models customarily used

Funding of this research by Spanish MINECO and the European FEDER Program (Projects MAT2011-22719 and ENE2011-29741) and by Gobierno de Aragón (Research group T12) is gratefully acknowledged.

A. Badía-Majós is with the Materials Science Institute of Aragón (ICMACSIC), Department of Condensed Matter Physics, University of Zaragoza, María de Luna 3, Zaragoza, E 50018 Spain (e-mail: anabadia@unizar.es).

A. Aliaga is with the Engineering and Architecture School, University of Zaragoza, María de Luna 3, Zaragoza, E 50018 Spain.

J. Letosa is with the Department of Electrical Engineering, University of Zaragoza, María de Luna 3, Zaragoza, E 50018 Spain.

M. Mora is with the Materials Science Institute of Aragón (ICMA-CSIC), Department of Material Science, University of Zaragoza, María de Luna 3, Zaragoza, E 50018 Spain

J. Peña is with the Engineering and Architecture School, University of Zaragoza, María de Luna 3, Zaragoza, E 50018 Spain. in the following terms: (i) Meissner limit models, (ii) frozenfield models [3], [4], and (iii) critical state models [5]. Only the latter may accurately describe the full set of relevant facts of magnetic levitation with type-II superconductors, including vertical and lateral forces, finite size effects and hysteretic behavior. A lot of work has been done along this line, and remarkably, one may find a number of publications that show a very good agreement between theory and experiment [6]-[10]. Some of these models are applied to experimental situations that allow a 2D approximation, and others are stated as $3 \mathrm{D}$, owing to the finite size characteristics of the systems under consideration. Certainly, together with an increased predictive power, the degree of complexity of $3 \mathrm{D}$ models is much higher. Nevertheless, as noticed in Ref. [2], one may identify a considerable number of practical situations that are very reasonably simulated in $2 \mathrm{D}$. In particular, the axially and translationally symmetric geometries fit the cases of (i) rotating bearings, flywheels and motors, and (ii) transportation systems. Still, analytical approximations have to be abandoned for quantitative purposes, and more or less intricate numerical modeling is still due. In fact, although some efforts have to be mentioned [11], no analytical model is able to capture all the facts described above in levitation experiments.

This work aims to supply the simplest possible tools for the simulation of 2D systems with the highest generality. The two mentioned geometries will be considered in a unified treatment. As a final result for the end-user, we will introduce an undemanding formalism that states the electromechanic response of the levitating machine by using a few lines of computer code, relating the main physical quantities to straightforward multiplications. The proposal relies on the knowledge of two material parameters, the so-called critical current density $J_{c}$ for the superconductor and the remnant magnetization $M_{0}$ for the accompanying permanent magnet, and takes the form of a finite-element-like approach based on the use of scalar functions on a 2D mesh.

The paper is organized as follows. First, in (Sec III), we describe the theoretical framework of our investigation. We establish the quasistatic approximation of the Maxwell equations for the hybrid superconductor/magnet system. Then, taking advantage of the symmetries, we introduce the "scalar" formulation of the problem and eventually propose a numerical method for obtaining the solution in a given process. The method, that involves a discretized version of the electromagnetic problem is described in detail in Sec II-B. It is basically built upon the concept of mutual inductance between electric circuits. Specifically, we show that the Maxwell equations, the energy of the system and the electromagnetic forces may all 
be written in terms of mutual inductance matrices. Motivated by the interest of evaluating the stiffness of maglev machines against arbitrary perturbations, in Sec II-B4 we concentrate on the response of the system to small displacements along the vertical and horizontal directions. The theory is validated against experiments in our test machine in Sec III

\section{2D MODELING OF LEVITATING EXPERIMENTS}

\section{A. Continuum electromagnetics: the MQS limit}

We start by establishing the range of application. Customarily, one can argue that, owing to the typical operation frequencies (small enough as compared to the superconducting material response) a magneto-quasi-static (MQS) approximation may be used. Then, the set of electromagnetic equations to consider may be cast as

$$
\begin{aligned}
& \operatorname{curl} \mathbf{H}=\mathbf{J} \equiv J \hat{\jmath} \\
& \operatorname{curl} \mathbf{E}=-\mu_{0} \frac{\partial \mathbf{H}}{\partial t} \Leftarrow \mathbf{E}=-\frac{\partial \mathbf{A}}{\partial t} \\
& \mathbf{E}(J)= \begin{cases}0 & , \quad J \leq J_{c} \\
\rho_{\mathrm{ff}}\left(J-J_{c}\right) \hat{\jmath} & , \quad J>J_{c}\end{cases}
\end{aligned}
$$

with $\hat{\jmath}$ the unit vector along the current density at each point of the superconductor, and $\rho_{\mathrm{ff}}$ the material property (resistivity) that may acquire an involved tensorial character [12], [13]. The second equation means that Faraday's law may be enforced by the use of the magnetic vector potential. A possible additional electrostatic term $\left(\mathbf{E}_{\Phi}=-\operatorname{grad} \Phi\right)$ could be absorbed by gauge transformation $\mathbf{A}^{\prime} \rightarrow \mathbf{A}=\mathbf{A}^{\prime}+\operatorname{grad} \chi$ with $\Phi=\partial \chi / \partial t$. As charge accumulation is negligible in the range of frequencies involved, ours is a natural selection for the potential, and one must not care about $\Phi$. It is apparent that the third equation models the superconducting behavior. Lossless current flow occurs below the critical threshold $J=J_{c}$, whereas dissipation appears beyond such value. The approximation that the resistivity $\rho_{\mathrm{ff}}$ acquires a very high magnitude leads to the critical state regime description [5], then, excursions of $J$ beyond $J_{c}$ are nearly instantaneous processes, controlled by the diffusion time constant $\tau \approx 1 / \rho_{\text {ff }}$.

The behavior of the permanent magnet may be introduced in a variety of forms. Here, assuming uniformity, we will use the effective magnetic current density, i.e.:

$$
\mathbf{K}_{\mathrm{M}}=\mathbf{M}_{0} \times \hat{\mathbf{n}}
$$

locally, at the magnet's lateral surface.

Thus, the MQS description of the electromagnetic problem needs to incorporate the fields $\mathbf{H}, \mathbf{J}, \mathbf{A}$ coupled by the above equations.

Though seemingly simple, the system (1) may be tough to solve for arbitrary geometries. Even the powerful Finite Element Methods developed for electromagnetic problems are seriously hampered by the superconducting law $\mathbf{E}(\mathbf{J})$, due to the complication introduced by the unknown dependence of $\hat{\boldsymbol{j}}(x, y, z)$. Let us pave the way for an undemanding method, by exploiting some symmetry properties.
1) Two dimensional statements for the fields $\mathbf{H}, \mathbf{J}, \mathbf{A}$ : We will discuss the 2D implementations that emerge from the symmetries applying to the cases of rotating machines or long transportation lines. In both cases $\hat{\jmath}$ is known a priori and $\rho_{\mathrm{ff}}$ becomes a scalar.

a) Translational symmetry.: Let us assume that the maglev system takes the form of a long guideway along the $y$ axis, such that the magnet is uniformly magnetized (for instance, along the $z$-axis): $\mathbf{M}_{0}=M_{0} \hat{\mathbf{z}}$, and is shaped as a long bar of rectangular cross section. Let its dimensions be $2 a \times h_{M}$ in the cross section ( $x z$ plane). The superconductor is also a long bar stretching along the $y$-axis and with cross section $2 b \times h_{S}$. If the superconductor is homogeneous and isotropic, the electromagnetic fields will take the form

$$
\begin{array}{r}
\mathbf{H}(x, z)=H_{x}(x, z) \hat{\mathbf{x}}+H_{z}(x, z) \hat{\mathbf{z}} \\
\mathbf{J}(x, z)=J_{y}(x, z) \hat{\mathbf{y}} \equiv J(x, z) \hat{\mathbf{y}} \\
\mathbf{A}(x, z)=A_{y}(x, z) \hat{\mathbf{y}} \equiv \Psi_{\mathbf{y}}(x, z) \hat{\mathbf{y}}
\end{array}
$$

A relevant consequence of these equations is that, owing to the symmetry, the general formulation of Eq. (1) greatly simplifies. In particular, as regards, the potential $\mathbf{A}$, one may forget about vectorial properties, because its orientation is determined. Thus, we stress the fact that $\left(J, \Psi_{\mathbf{y}}\right)$ are the two basic scalar fields of the problem. In particular, by using $\mu_{0} \mathbf{H}=\operatorname{curl} \mathbf{A}$ one can write [14]

$$
\begin{aligned}
\mu_{0} H_{x} & =-\frac{\partial \Psi_{\mathbf{y}}}{\partial z} \\
\mu_{0} H_{z} & =\frac{\partial \psi_{\mathbf{y}}}{\partial x}
\end{aligned}
$$

Related to these equations, recall that a so-called 2D "streamfunction formulation" is possible through the straightforward relation

$$
\mu_{0} \mathbf{H}=\operatorname{grad}_{2} \Psi_{\mathbf{y}} \times \hat{\mathbf{y}},
$$

where $\operatorname{grad}_{2}$ means the 2D operator $\left(\partial_{x}, \partial_{z}\right)$ and $\Psi_{\mathbf{y}}$ plays the role of a magnetostatic potential. Among other useful properties, this means that the contour lines of $\Psi_{\mathbf{y}}$ are nothing but the field lines of $\mathbf{H}$. This follows from

$$
d \Psi_{\mathbf{y}}=d \mathbf{s} \cdot \operatorname{grad}_{2} \Psi_{\mathbf{y}}=0 \Rightarrow \frac{d s_{x}}{d s_{z}}=\frac{-\partial_{z} \Psi_{\mathbf{y}}}{\partial_{x} \Psi_{\mathbf{y}}}=\frac{H_{x}}{H_{z}},
$$

where $d \mathbf{s}$ stands for a generic displacement along the "constant potential" lines $\left(\Psi_{\mathbf{y}}=\right.$ constant $)$.

Also, one can see that solenoidality condition $\operatorname{div} \mathbf{H}=0$ is authomatically enclosed in Eq. (5).

We want to emphasize that, by using Ampère's law, the full electromagnetic problem could be stated in terms of $\Psi_{\mathbf{y}}$ and its derivatives, supplied with appropriate boundary conditions. However, aiming at the technical simplicity of the eventual numerical problem, we will prefer a mixed statement $\left(\Psi_{\mathbf{y}}, J\right)$ formulation. More specifically, in the case of the magnetic source, the magnetization currents will be given, and the streamfunction obtained from them. For the superconductor, $J$ will be unknown, and the evaluation of $\Psi_{\mathbf{y}}$ will be done after solving the problem. In fact, at the numerical level, we will take advantage of the linear relation between currents 
and "potentials". Thus, the potential created at the point $\mathbf{r}=(x, y, z)$ by a long wire that settles at $\left(x_{i}, z_{i}\right)$ reads

$$
\Psi_{\mathbf{y}, i}(\mathbf{r})=\frac{\mu_{0} I_{i}}{4 \pi} \int \frac{d y_{i}}{\sqrt{\left(x-x_{i}\right)^{2}+y_{i}^{2}+\left(z-z_{i}\right)^{2}}}+\mathcal{C} .
$$

Recall that we have included a constant $\mathcal{C}$ that will be used to deal with the artificial divergences arising from the infinite wire approximation.

b) Rotational symmetry: we proceed by showing that a full analogy exists for the case of rotational symmetry: one may also define a scalar field formulation in a Euclidean 2D space. Here we assume that the levitating machine consists in a cylindrical magnet coaxial with a superconducting cylinder. We will denote their respective radii by $b$ and $a$, and their heights by $h_{M}$ and $h_{S}$. Let us start by using the cylindrical coordinate system, in which the electromagnetic fields read

$$
\begin{aligned}
\mathbf{H}(\rho, z) & =H_{\rho}(\rho, z) \hat{\boldsymbol{\rho}}+H_{z}(\rho, z) \hat{\mathbf{z}} \\
\mathbf{J}(\rho, z) & =J_{\varphi}(\rho, z) \hat{\boldsymbol{\varphi}} \equiv J(\rho, z) \hat{\boldsymbol{\varphi}} \\
\mathbf{A}(\rho, z) & =A_{\varphi}(\rho, z) \hat{\boldsymbol{\varphi}}
\end{aligned}
$$

Again, it is apparent that the vector potential is not necessarily given as vector quantity. Thus, starting with $\mu_{0} \mathbf{H}=\operatorname{curl} \mathbf{A}$ one obtains

$$
\begin{aligned}
& \mu_{0} \rho H_{\rho}=-\frac{\partial}{\partial z}\left(\rho A_{\varphi}\right) \equiv-\frac{\partial \Psi_{\varphi}}{\partial z} \\
& \mu_{0} \rho H_{z}=\frac{\partial}{\partial \rho}\left(\rho A_{\varphi}\right) \equiv \frac{\partial \Psi_{\varphi}}{\partial \rho}
\end{aligned}
$$

Now, the "streamfunction" formulation that generates the field becomes:

$$
\mu_{0} \mathbf{H}=\frac{1}{\rho} \operatorname{grad}_{2} \Psi_{\varphi} \times \hat{\varphi}
$$

For the readers' sake we mention that this function is well known in fluid mechanics and named after "Stokes streamfunction" related to the problem of incompressible three dimensional axisymmetric flows. For our purposes, a couple of properties must be highlighted. On the one side, the contour lines of $\Psi_{\varphi}$ are also the streamlines of $\mathbf{H}$ :

$$
d \Psi_{\varphi}=d \mathbf{s} \cdot \operatorname{grad}_{2} \Psi_{\varphi}=0 \Rightarrow \frac{d s_{\rho}}{d s_{z}}=\frac{-\partial_{z} \Psi_{\varphi}}{\partial_{\rho} \Psi_{\varphi}}=\frac{H_{\rho}}{H_{z}}
$$

On the other side, as in the translational symmetry, one can also take advantage of the linear relation between currents and potentials. Now, the potential created at a given point $\mathbf{r}$ by a circular current loop, carrying $I_{i}$ is:

$$
\Psi_{\varphi, i}(\mathbf{r})=\frac{\mu_{0} I_{i}}{2 \pi} \oint_{C} \oint_{C^{\prime}} \frac{d \mathbf{l} \cdot d \mathbf{l}_{i}}{\left\|\mathbf{r}-\mathbf{r}_{i}\right\|}
$$

with the definitions

$\mathbf{r}_{i}$ : generic point at the source circular current loop.

$d \mathbf{l}$ : differential vector tangent along the circle $C$ that includes the field point $\mathbf{r}$.

$d \mathbf{l}_{i}$ : differential vector tangent along the circle $C^{\prime}$ that includes the source point $\mathbf{r}_{i}$

We call the readers' attention that the above expression closely resembles the Neumann formula for mutual inductances, a fact that will be advantageous below.
To conclude this section, we want to emphasize that the 2D problems exposed above are formally equivalent. Thus, hereafter, we can use a common notation $(J(x, y), \Psi(x, y))$ for both cases. Just, one has to recall that for the cylindrical symmetry, $x$ plays the role of $\rho$, and recall the "metric" factor $1 / \rho$ when deriving $\mathbf{H}$ from $\Psi$.

2) The Maxwell equations in the MQS limit. Variational statement: Having defined the electromagnetic field structure of our problem, we now proceed by introducing an alternative formulation for the Maxwell equations in the MQS limit (Eq.(1)). A variational statement will be preferred to the conventional differential equation form, mainly motivated by the convenience for the numerical treatment when superconducting materials are involved. Considering that the basic unknown quantity in the statement will be the local supercurrent density, one gets the benefit of: (i) avoiding to implement involved boundary conditions for the fields and (ii) increase the stability of calculations because the quantities of interest are obtained by integration [15].

The main concepts were introduced in Ref. [16]. In brief, the process of magnetic diffusion that takes place when the bias is modified, obeys the variational statement $\min \mathcal{F}[\mathbf{J}(\mathbf{r})]$, with $\mathcal{F}$ the functional given by

$$
\begin{aligned}
\mathcal{F}[\mathbf{J}]= & \frac{\mu_{0}}{4 \pi} \iint_{\Omega}\left[\frac{1}{2} \frac{\mathbf{J}(\mathbf{r}) \cdot \mathbf{J}\left(\mathbf{r}^{\prime}\right)-2 \mathbf{J}_{0}(\mathbf{r}) \cdot \mathbf{J}\left(\mathbf{r}^{\prime}\right)}{\left\|\mathbf{r}-\mathbf{r}^{\prime}\right\|}\right] d V d V^{\prime} \\
& +\int_{\Omega} \Delta \mathbf{A}_{\mathrm{S}} \cdot \mathbf{J} d V+\Delta t \int_{\Omega} \mathcal{P}[\mathbf{J}(\mathbf{r})] d V
\end{aligned}
$$

Here, the following notation has been used:

$\Omega$ : region occupied by the superconductor

$\mathbf{J}(\mathbf{r})$ : (unknown) current density at point $\mathbf{r}$ and incremented time $t_{0}+\Delta t$

$\mathbf{J}_{0}(\mathbf{r})$ : (given) current density at $\mathbf{r}$ and previous time $t_{0}$ $\Delta \mathbf{A}_{S}$ : variation of the vector potential created by the sources (the magnet in our case) along $\Delta t$

$\mathcal{P}[\mathbf{J}]$ : power dissipated per unit volume at a given point within the superconductor

For further application it will be useful to consider each term of the functional by separate, and thus we define

$$
\mathcal{F} \equiv \mathcal{F}_{\mathrm{JJ}}+\mathcal{F}_{\mathrm{J} 0}+\mathcal{F}_{\mathrm{JS}}+\Delta t \mathcal{W}_{\mathrm{JE}}
$$

with

$\mathcal{F}_{\mathrm{JJ}}$ : self energy of the evolutionary circulating currents $\mathcal{F}_{\mathrm{J} 0}$ : interaction energy of the evolutionary currents with a frozen distribution

$\mathcal{F}_{\text {JS }}$ : interaction energy of the evolutionary currents with the magnetic source

$\Delta t \mathcal{W}_{\mathrm{JE}}$ : energy related to the entropy production due to dissipative mechanisms

Eq. (13) is a powerful statement for solving quasi-steady conduction problems. One may trace the evolution $\mathbf{J}(\mathbf{r}, t)$ for any given material dissipation law $\mathcal{P}$ and excitation process (say $\mathbf{A}_{\mathrm{S}}(\mathbf{r}, t)$ ). For instance, $\mathcal{P}=\rho_{0} J^{2} / 2$ would portrait the behavior of a normal conducting sample of resistivity $\rho_{0}$. Specific dependencies $\mathcal{P}(\mathbf{J})$ for hard superconductors (as the one related to the law in Eq.(1) have been discussed in many works. Corresponding to the quasi-linear $\mathbf{E}(\mathbf{J})$ expression in 


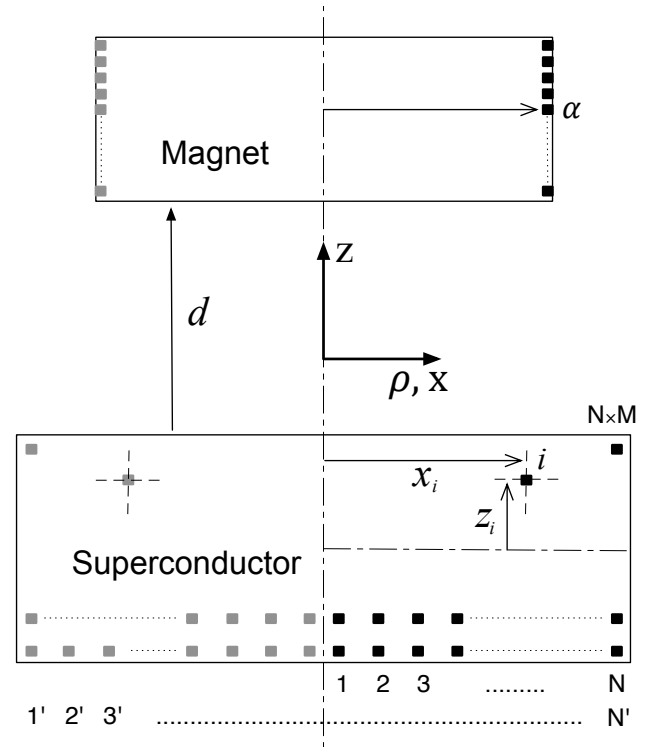

Fig. 1: Sketch of the interacting magnet and superconductor in 2D geometry. The discretization of the system into elementary current circuits is indicated. The superconductor needs a two dimensional grid of circuits that are named after latin indices. The divisions for rotational $(N \times M)$ and translational geometries $\left(N^{\prime} \times M\right)$ are indicated.

Eq.(1), and for the 2D problems, the related dissipation term takes the form

$$
\mathcal{P}_{\mathrm{JE}}= \begin{cases}0 & \text { if }|J| \leq J_{c} \\ (1 / 2) \rho_{\mathrm{ff}}\left(J-J_{c}\right)^{2} & \text { if } J>J_{c} . \\ (1 / 2) \rho_{\mathrm{ff}}\left(J+J_{c}\right)^{2} & \text { if } J<-J_{c} .\end{cases}
$$

Notice that $\rho_{\mathrm{ff}}$ is the material parameter that describes the sharpness of the transition from the superconducting state to dissipation, when the threshold $J_{c}$ is reached. In many practical situations, transitions are rather steep and the induced electric fields are such that $E_{0} / \rho_{\mathrm{ff}} J_{c}$ is a small parameter. This means that $J$ goes very little beyond $J_{c}$ and the term $\Delta t \mathcal{W}_{\mathrm{JE}}$ may be just replaced by the critical state condition $|J| \leq J_{c}$, holding "quasistatically" along the time [12].

\section{B. Discrete formulation}

As numerical techniques will be used, one needs to translate the previous equations to the language of discrete systems. Here, this implies to define a virtual collection of circuits that will carry the unknown superconducting current distribution. Also, the magnetic sources of the problem (cylindrical or bar shaped magnets with uniform magnetization) may be pictured as a collection of surface currents $\left(\mathbf{K}_{\mathrm{M}}=\mathbf{M}_{0} \times \hat{\mathbf{n}}\right)$. Below, we describe the basic geometrical concepts of the ensemble: location and labeling, mutual inductance, energy of the system, forces, etc. As shown in Fig 1 the collection of circuits (either long straight wires or coaxial rings) may be mapped by using a square grid. Each point of the grid (labelled with latin index $i$ in the superconductor, and with the greek $\alpha$ for the magnet, will be allocated according to the normalized cartesian coordinates:

$$
\begin{aligned}
\tilde{x}_{i} & \equiv \frac{x_{i}}{\delta}=i+N\left(1-\left\lceil\frac{i}{N}\right\rceil\right)+X_{S 0} \\
\tilde{z}_{i} & \equiv \frac{z_{i}}{\delta}=\left\lceil\frac{i}{N}\right\rceil+Z_{S 0} \\
\tilde{x}_{\alpha} & \equiv \frac{x_{\alpha}}{\delta}=X_{M 0} \\
\tilde{z}_{\alpha} & \equiv \frac{z_{\alpha}}{\delta}=\alpha+Z_{M 0}
\end{aligned}
$$

Here, $\delta$ is the unit cell spacing and we have used the standard notation for the ceiling functions, i.e.: $\lceil z\rceil=\min \{n \in \mathbb{Z} \mid n \geq$ $z\}$. On the other hand, $X_{S 0}, Z_{S 0}$ and $X_{M 0}, Z_{M 0}$ are constant values to be adjusted according to the position wanted for the origin of coordinates.

1) Circuits and inductances: Mutual inductance is a central concept in the discrete resolution of our problem. Related to this, a number of specialized treatises [17] provide a collection of useful formulas. Thus, the mutual inductance between two circular loops $i$ and $j$, respectively described by the coordinates $\left(x_{i}, z_{i}\right)$ and $\left(x_{j}, z_{j}\right)$ in our scheme, is given by

$$
M_{i j}^{\circ}=\mu_{0} \sqrt{x_{i} x_{j}}\left[\left(\frac{2}{k}-k\right) K(k)-\frac{2}{k} E(k)\right]
$$

with the definition

$$
k=\sqrt{\frac{4 x_{i} x_{j}}{\left(x_{i}-x_{j}\right)^{2}+\left(z_{i}-z_{j}\right)^{2}}}
$$

and $K(k), E(k)$ the complete elliptic integrals of the first and second kind. The self inductance of the loops is given by

$$
M_{i i}^{\circ}=\mu_{0} x_{i}\left[\ln \frac{x_{i}}{\delta}+0.9018\right] .
$$

On the other hand, for the case of long wires, one can find the following expressions that correspond to the unit length inductance coefficient for wires of radius $a$

$$
\begin{aligned}
M_{i j}^{\|} & =\frac{\mu_{0}}{2 \pi} \ln \frac{a^{2}}{\left(x_{i}-x_{j}\right)^{2}+\left(z_{i}-z_{j}\right)^{2}} \\
M_{i i}^{\|} & =\frac{\mu_{0}}{8 \pi}
\end{aligned}
$$

For further use, we introduce the dimensionless inductance coefficient in 2D problems (valid for both geometries):

$$
m_{i j} \equiv M_{i j}\left(\tilde{x}_{i}, \tilde{x}_{j}, \tilde{z}_{i}, \tilde{z}_{j}\right) / \mu_{0}
$$

\section{2) Energies and forces in $2 D$ problems:}

a) Free energy of the system: The reader may straightforwardly check that the free energy terms in Eq. 113 ) are evaluated as follows in discretized form (just recall the Neumann formula for mutual inductances)

$$
\begin{gathered}
\mathrm{F}_{\mathrm{JJ}}=\frac{\mu_{0}}{2} J_{c}^{2} \delta^{5} \sum_{i j} \jmath_{i} m_{i j} \jmath_{j} \\
\mathrm{~F}_{\mathrm{J} \mathrm{O}}=-\mu_{0} J_{c}^{2} \delta^{5} \sum_{i j} \check{\jmath}_{i} m_{i j} \jmath_{j} \\
\mathrm{~F}_{\mathrm{JS}}=\mu_{0} J_{c}^{2} \delta^{5} \sum_{i} \Delta \psi_{i}^{P} \jmath_{i}
\end{gathered}
$$


In these equations, we have introduced dimensionless units for the current density, in terms of the material critical current density, i.e.: $\jmath_{i} \equiv J\left(x_{i}, y_{i}\right) / J_{c}$. Tilded quantities “ $\sim$ mean the given values for the previous time $\left(\check{\jmath}_{i} \equiv \jmath_{i}\left(t_{0}\right)\right)$. The vector potential $\mathbf{A}_{\mathrm{S}}$ in the interaction term $\mathcal{F}_{\mathrm{JS}}$ has been replaced by the streamfunction in the discrete version. Thus, $\psi_{i}^{P}$ "represents" the vector potential created by the magnetic sources at the region of the superconducting circuit $i$ and $\Delta \psi_{i}^{P} \equiv \psi_{i}^{P}-\check{\psi}_{i}^{P}$.

$\psi_{i}^{P}$ can be evaluated by using the linear property announced in Eqs. (7) and (12) and recalling the discretized expression for the magnetization currents

$$
I_{\alpha}=K_{M, \alpha} \delta=M_{0} \delta
$$

with $I_{\alpha}$ standing for a volume current density at the circuit $\alpha$

Then, one gets the normalized streamfunction

$$
\psi_{i}^{P} \equiv \frac{\Psi\left(\mathbf{r}_{i}\right)}{\mu_{0} J_{c} \delta^{2}}=P \sum_{\alpha} m_{\alpha i}
$$

with $P$ the dimensionless parameter that accounts for the "relative strength" of the magnet and the superconductor

$$
P \equiv \frac{M_{0}}{J_{c} \delta}
$$

and $m_{\alpha i}$ the mutual inductance coefficient between the magnetic circuit $\alpha$ and the superconducting circuit $i$.

For further application, it is useful to note that the discrete version of the streamfunction related to the superconducting currents reads

$$
\psi_{j}^{s c} \equiv \psi^{s c}\left(\mathbf{r}_{j}\right)=\sum_{i} m_{j i} j_{i}
$$

with $\jmath_{i}$ the current density at the circuit $i$.

Thus, we get the following expression of the free energy function to be minimized, so as to obtain the updated configuration of supercurrents $\left\{J_{i}\right\}$ at $t_{0}+\Delta t$

$$
\mathrm{F}\left[\left\{J_{i}\right\}\right]=\frac{1}{2}\langle\mathrm{~J}|\mathrm{~m}| \mathrm{J}\rangle-\langle\check{\mathrm{J}}|\mathrm{m}| \mathrm{J}\rangle+\left\langle\Delta \psi^{P} \mid \mathrm{J}\right\rangle
$$

Here, we have defined the $g$ dimensional $(g=N \times M)$ vector quantities, formed by the values of the current density at the grid points, i.e.:

$$
|\mathrm{J}\rangle \equiv\left(\begin{array}{c}
\jmath_{1} \\
\jmath_{2} \\
\cdot \\
\cdot \\
\jmath_{g}
\end{array}\right) \quad, \quad\langle\mathrm{J}| \equiv\left(\jmath_{1}, \jmath_{2}, \ldots, \jmath_{g}\right)
$$

Also, for compactness, we are using Dirac's "ket" and "bra" notation, with $\langle\mathrm{J}|$ the transpose of $|\mathrm{J}\rangle$.

From the technical point of view, the mathematical problem to be solved (find the $g$-vector $|\mathrm{J}\rangle$ that minimizes $\mathrm{F}\left[\left\{J_{i}\right\}\right]$ subject to the constraints $-1<j_{i}<1, i=1,2, \ldots g$ ) is a large non-linear constrained optimization problem. Accurate simulations will typically mean $g$ values around several thousands. Noteworthily, Eq. 27) fits the so-called quadratic programming paradigm. This very well known problem has received much attention between applied mathematicians, and the interested reader may benefit from an abundant collection of excellent software solutions. For instance, the popular languages OCTAVE and MATLAB ${ }^{\circledR}$ implement the quadratic problem by means of the specific built-in functions $\mathrm{qp}()$ and quadprog() respectively. The user must plainly input the values of the matrix $\mathrm{m}$ and the arrays $\left|\psi^{P}\right\rangle,|\breve{\mathrm{J}}\rangle$. Thus, one may solve the evolutionary critical state statement of the levitation problem by iteration of the single line command

$$
|\mathrm{J}\rangle:=\mathrm{QP} \_ \text {function }\left(\mathrm{m},\left|\mathrm{A}_{0}\right\rangle,\left|\mathrm{L}_{b}\right\rangle,\left|\mathrm{U}_{b}\right\rangle, \mathrm{T}\right)
$$

Here, we have defined $\left|\mathrm{A}_{0}\right\rangle=\left|\psi^{P}\right\rangle-\left|\check{\psi}^{P}\right\rangle-\mathrm{m}|\check{\mathrm{J}}\rangle$ and the critical current bounds, that for isotropic conditions read

$$
\left|\mathrm{L}_{b}\right\rangle \equiv\left(\begin{array}{c}
-1 \\
-1 \\
\cdot \\
\cdot \\
-1
\end{array}\right) \quad, \quad\left|\mathrm{U}_{b}\right\rangle \equiv\left(\begin{array}{c}
1 \\
1 \\
\cdot \\
\cdot \\
1
\end{array}\right)
$$

$\mathrm{T}$ represents the transport current condition. In our case, simply $\mathrm{T}=\sum \jmath_{i}=0$. $\mathrm{QP} \_$function stands for either $\mathrm{qp}()$ or quadprog () in the mentioned instances. Both options have been tested successfully, checked against the powerful optimization fortran package LANCELOT [18].

b) Forces: Being able to obtain the solution $|J\rangle$ for an arbitrary configuration, one may readily evaluate the mechanical behavior of the system. Thus, performing "virtual displacements" around a given position, forces arise as partial derivatives of the interaction energy. For instance, the normalized force acting on the superconductor due to the interaction with the magnet may be obtained from

$$
\mathbf{f}=\operatorname{grad}_{2}\left\langle\psi^{P} \mid \mathrm{J}\right\rangle=\left\langle\operatorname{grad}_{2} \psi^{P} \mid \mathrm{J}\right\rangle .
$$

This equation is the counterpart of the celebrated Lorentz force expression $\mathbf{F}=\mu_{0} \int \mathbf{J} \times \mathbf{H} d V$, from which it can be obtained just by replacing $\mu_{0} \mathbf{H}=\operatorname{grad}_{2} \psi \times \hat{\mathbf{u}}$ and applying vector algebra. Here, our interpretation is that it defines the "force projector", that allows to obtain the force components for any given configuration $\left\langle\operatorname{grad} \psi^{P}\right|$. In practice:

$$
\operatorname{grad}_{2} \psi_{i}^{P}=P \sum_{\alpha} \operatorname{grad}_{2} m_{\alpha i} \equiv P \sum_{\alpha} \mathbf{n}_{\alpha i}
$$

Notice that the bold notation indicates the vectors in real space, not to be confused with the $\left\{J_{i}\right\}$ components of the current density in the "finite element space". For example, if one assumes a vertical displacement of the magnet in the cylindrically symmetric system, the matrix elements $n_{\alpha i}^{z}$ are given by

$$
\begin{aligned}
n_{\alpha i}^{z}= & \frac{z_{\alpha}-z_{i}}{\sqrt{\left(x_{\alpha}+x_{i}\right)^{2}+\left(x_{\alpha}+x_{i}\right)^{2}}}\left[K\left(k_{i}\right)\right. \\
& \left.-\frac{x_{\alpha}^{2}+x_{i}^{2}+\left(z_{\alpha}-z_{i}\right)^{2}}{\left(x_{\alpha}-x_{i}\right)^{2}+\left(z_{\alpha}-z_{i}\right)^{2}} E\left(k_{i}\right)\right]
\end{aligned}
$$




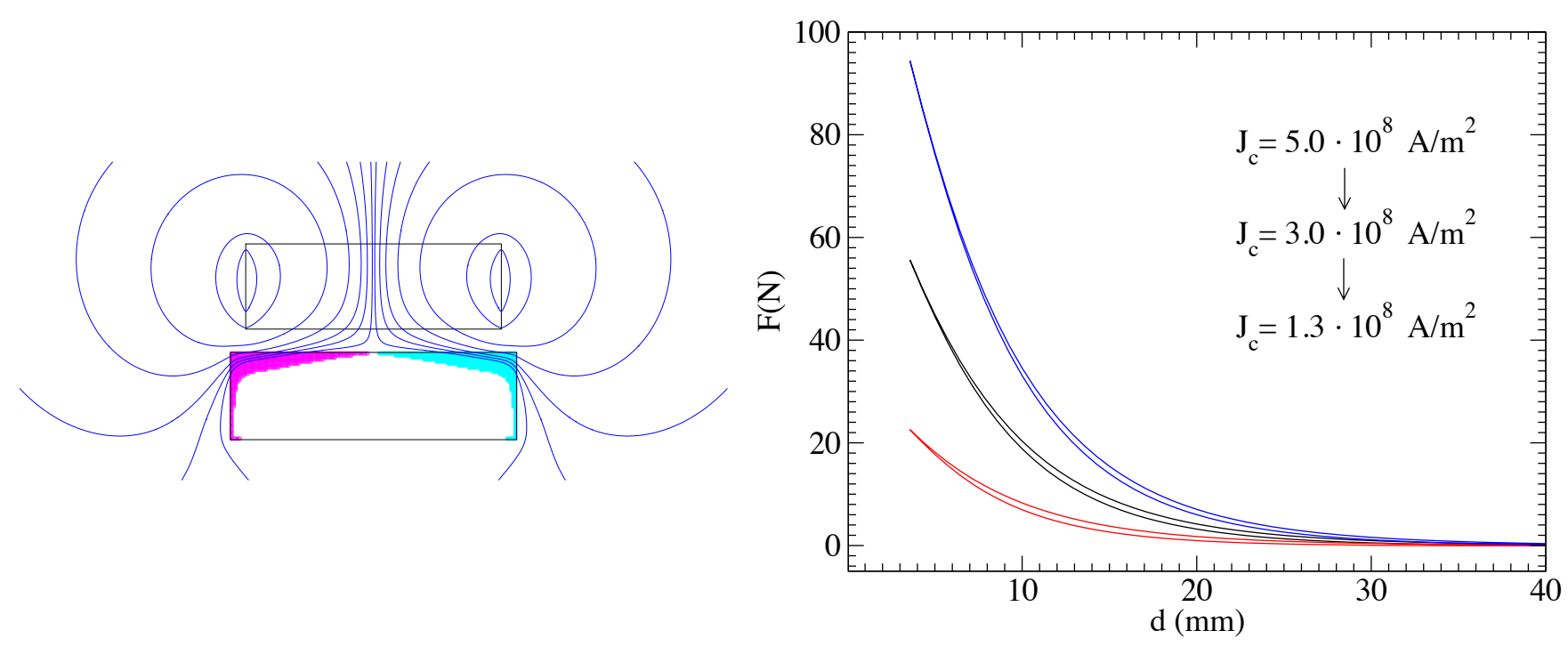

Fig. 2: Left: magnetic field lines around a cylindrical magnet/superconductor arrangement. Also indicated are the penetrating supercurrent circular loops. To the right, we show full hysteresis cycles of the vertical force for different values of the critical current density parameter in the ZFC case.

with the definition

$$
k_{i}=\sqrt{\frac{4 x_{\alpha} x_{i}}{\left(x_{\alpha}+x_{i}\right)^{2}+\left(z_{\alpha}-z_{i}\right)^{2}}},
$$

and the levitation forces read

$$
f_{z}=P \sum_{i, \alpha} n_{\alpha i}^{z} j_{i}
$$

Apparently, by using this equation, one obtains the force in dimensionless units. It may be rescaled to physical units by

$$
F_{z}=\mu_{0} J_{c}^{2} \delta^{4} f_{z}
$$

3) Application to rotationally symmetric problems: In this section, we consider the case of a cylindrical magnet/superconductor arrangement. As usual, Field cooling (FC) and Zero Field Cooling (ZFC) conditions were simulated by selecting the initial position of the magnet and plainly implying the initial zero current condition $\left|J_{0}\right\rangle=0$ for the superconductor.

Fig 2 illustrates the results for the specific set of conditions:

- Magnet: $\mu_{0} M_{0}=1.17 \mathrm{~T}, h_{M}=15 \mathrm{~mm}, 2 b=45 \mathrm{~mm}$.

- Superconductor: various values of $J_{c} \approx 10^{8} \mathrm{~A} / \mathrm{m}^{2}, h_{S}=$ $15 \mathrm{~mm}, 2 a=50 \mathrm{~mm}$.

These correspond to our home-made levitation assembly, built with standard commercial materials (MT-YBaCuO superconducting cylinder and $\mathrm{NdFeB}$ permanent magnet).

The superconducting region was meshed with a rectangular grid of $70 \times 42=3010$ elements, each representing a circular loop of current. Minimization of Eq.27) was performed for a number of vertical trajectories of the magnet, step by step, with increments of $0.2 \mathrm{~mm}$.

Fig 2 displays the magnetic field structure induced by cooling the superconductor with the magnet at a big enough distance (in practice $d=60 \mathrm{~mm}$ suffices) so as to approach the ZFC condition. Also we show the full hysteresis cycle for the force subsequent to the descending/ascending trajectory.

For each position of the magnet along the path (that we parameterize by the time values $\left.t_{n}\right)$, we get $\left|J\left(t_{n}\right)\right\rangle \equiv$ $\left(\jmath_{1 n}, \jmath_{2 n} \cdots \jmath_{g n}\right)$, and with this, we have been able to evaluate the "superconducting" streamfunction at any position of space $\left(\mathbf{r}_{j}\right)$ by using Eq. 26. Then, as follows from Eq. 111 one can plot the magnetic field lines just by drawing the contour lines of $\Psi_{n}^{f u l l}=\Psi_{n}^{s c}+\Psi_{n}^{P}$. Combined with the built-in function contour() this was done to obtain the left panel of Fig 2 On the other hand, the calculation of the levitation force is a straightforward application of Eq. (34). At the step of time $t_{n}$ one has

$$
f_{n, z}=\left\langle\partial_{z} \Psi_{n}^{P} \mid J_{n}\right\rangle=P \sum_{i, \alpha} n_{\alpha i}^{z} J_{i n}
$$

This equation, with $n_{\alpha i}^{z}$ replaced by the expression in Eq. 32, leads to the results of the right pane of Fig 2 . They were obtained for standard values of $J_{c}$ in MT-YBaCuO at $77 \mathrm{~K}$.

4) Application to translationally symmetric problems:

Here, we show that our model may provide useful information about the stability issues in levitation machines. We have investigated a system composed by a vertically magnetized bar-shaped permanent magnet parallel to a superconducting bar. The cross section of the system fits the schema in Fig 1 . with the material parameters:

- Magnet: $\mu_{0} M_{S}=1.17 \mathrm{~T}, h_{M}=15 \mathrm{~mm}, b$ variable.

- Superconductor: $J_{c}=3 \times 10^{8} \mathrm{~A} / \mathrm{m}^{2}, h_{S}=15 \mathrm{~mm}, 2 a=$ $50 \mathrm{~mm}$.

The superconducting region was meshed with a grid of $100 \times 30=3000$ elements, each representing a long wire. The magnet was described as a pile of 30 elementary wires at each side, standing for the magnetization currents. The starting point of our simulations was always taken in "centered" 

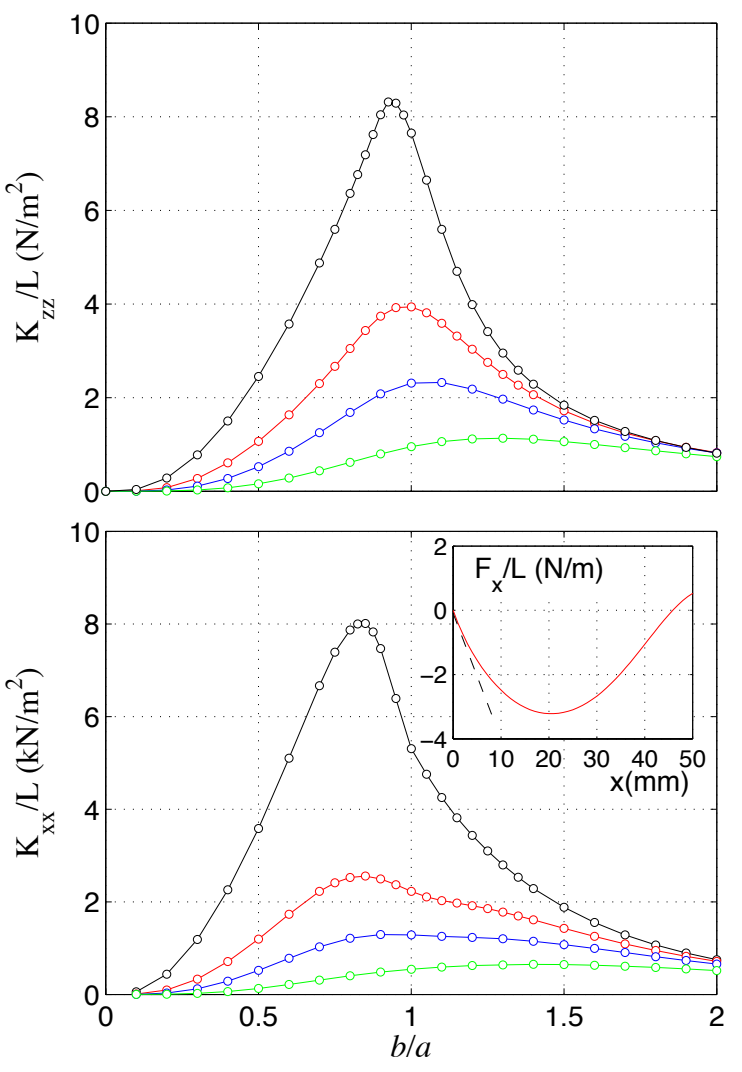

Fig. 3: Levitation $\left(K_{z z}\right)$ and guidance $\left(K_{x x}\right)$ stiffness coefficients dependence on the relative width $(b / a)$ of the magnet and superconductor. The different curves correspond to the cooling distances between them (from top to bottom) $d / a=$ $0,0.1,0.2,0.4$. In the inset we give a detail of the behavior of $F_{x}(x)$.

configuration. Then, two kinds of numerical experiments were performed so as to characterize the stiffness coefficients:

- "Vertical disturbances" were considered for obtaining $K_{z z}$. The linear regime was evaluated as the first millimeter displacement, for each cooling distance.

- "Horizontal disturbances" were also studied for obtaining $K_{x x}$. Again, the first millimeter provided a linear regime of $F_{x}(x)$.

We summarize our results in Fig 3 . The vertical (levitation) and lateral (guidance) stiffness coefficients are plotted against the relative dimensions of the magnet and the superconductor. Notice that, for the whole range of values, both $K_{x x}$ and $K_{z z}$ are positive, as corresponds to a stable system. More in detail, it is also clear that the maximum of $K_{z z}$ occurs close to $b / a=$ 1 (as between permanent magnets). The maxima of $K_{x x}$ for small values of the separation $d$ are clearly shifted to the left.

A feature to mention in the behavior of $K_{x x}$ is the "kink" that occurs at the value $b / a=1$, clearly visible for the lower distances. This is a finite size effect that one can understand by

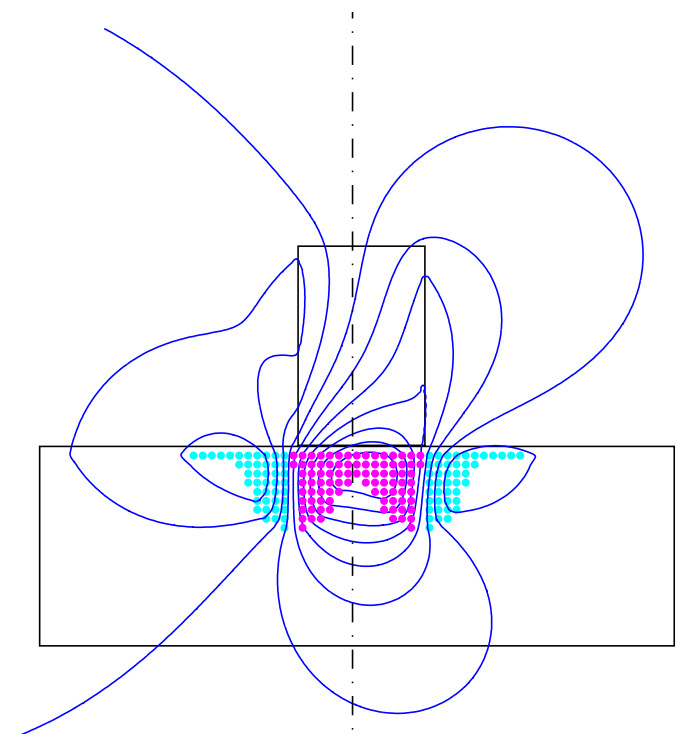

Fig. 4: Magnetic field lines obtained for a lateral displacement in the FC condition with $b / a=0.2$ and $d=0$.

plotting the field structure for the system as done in Fig 4 . For the range $0<b<a$ superconducting currents are not severely constrained by the size of the superconductor, and lateral current flow is not important. However, as $b$ increases, the superconducting currents stretch, and suddenly get constrained when the condition $b=a$ is reached.

For the readers' sake, we mention that a number features observed in Fig 3 agree with other results of the literature. First, field cooling at a non-zero distance produces a vertical stiffness two times greater than the horizontal, as described in experiments [4]. In fact, as noted by the authors of that paper, in their simplified analysis by image techniques, such property is related to the high critical current density of the system that makes the actual $J_{c}$ parameter irrelevant. In our case, the value of $J_{c}$ enters the theory, but as shown in Fig 2 it is high enough (recall the small hysteresis). Also, we note that the maximum value of $K_{z z}$ at $b / a \approx 1$ is a feature already reported by other authors that apply similar numerical techniques within the critical state formalism [19].

A technical issue regarding the identification of the initial slope of $\mathbf{F}$ must be clarified. We have evaluated the evolution of this quantity for small displacements $\delta x, \delta z$ around equilibrium. As illustrated in the inset of Fig 3 an initial "linear" behavior can be inferred. Quantitatively, a correlation coefficient $R>0.97$ was achieved for all the cases displayed, by considering the region mentioned: $\delta x, \delta z \lesssim 1 \mathrm{~mm}$.

\section{EXPERIMENTAL VALIDATION}

\section{A. Experimental setup}

The axial component of the levitation force between a bulk YBCO superconductor and a $\mathrm{NdFeB}$ permanent magnet (PM) was measured using the tension-compression tests in a universal test machine, INSTRON model 5565. The machine 

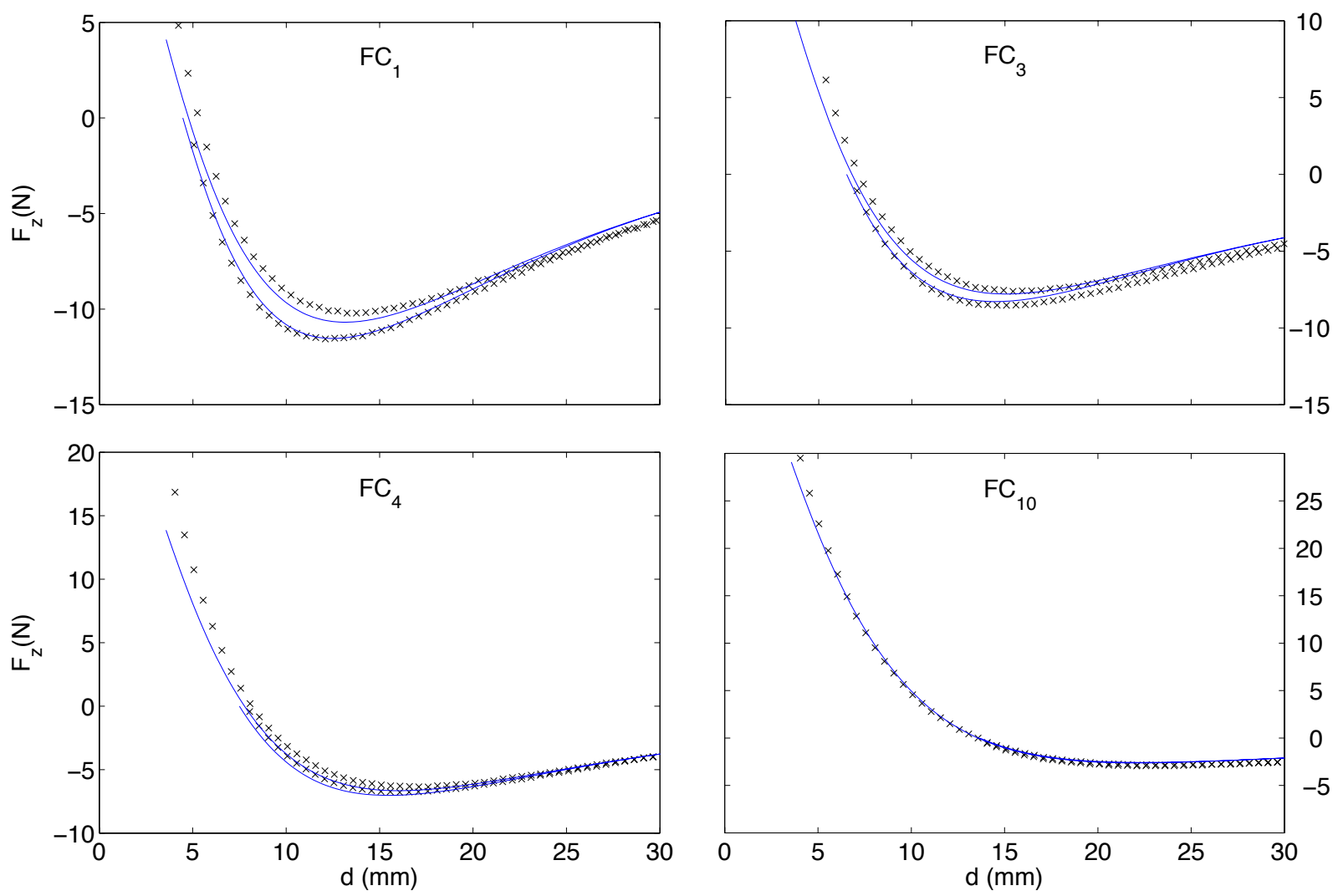

Fig. 5: Quantitative comparison between simulated and measured data on the cylindrical system. Continuous lines correspond to the theory and crosses to the experiment.

was equipped with a full electromechanical control and data acquisition via software. Bulk YBCO superconductor (Can Superconductors, CSYL-50) and NdFeB PM (Idemag, N35) with diameters and heights of $50 \mathrm{~mm}, 15 \mathrm{~mm}$ and $45 \mathrm{~mm}$, $15 \mathrm{~mm}$, respectively, were used in these measurements. The critical temperature of our YBCO sample is around $90 \mathrm{~K}$, while the remnant induction of the PM is $1.17 \mathrm{~T}$. The setup scheme basically fits Fig 1

The lower tensile grip of the universal test machine was replaced by a liquid nitrogen container, fixed to the tensile table. The superconductor was placed and fixed within this container, while the PM was attached to the bottom of a cylindrical aluminium holder, placed above the superconducting sample by matching their axes. The PM along with its support were attached to the upper tensile grip.

The axial component of the levitation force was measured as a function of the distance between the superconducting sample and the PM using $10 \mathrm{~mm} / \mathrm{min}$ displacement speed. The tests were performed by cooling down the YBCO superconductor to $77 \mathrm{~K}$ under the action of magnetic field provided by the PM (Field Cooling). Several cooling heights were investigated, with a minimum distance of $3.57 \mathrm{~mm}$, as determined by the thickness of the cryogenic container's cover.

\section{B. Experimental results}

1) Theory vs. experiment: Fig 5 shows the validation of our theory against the experiments performed in the setup described above. We display the hysteretic response of the levitation force corresponding to a set of field cooled experiments labelled $F C_{1}, F C_{3}, F C_{4}, F C_{10}$ as shown. In all cases, the superconductor was cooled with the magnet at the given height over the cryostat $(1,3,4,10 \mathrm{~mm}$ respectively). To start with, the vertical force was measured up to an ascending vertical distance of $60 \mathrm{~mm}$ (lower lines in the plots). Then, the descending branch was recorded, and the resulting plots correspond to the upper lines (when visible).

Notice that hysteresis is more and more pronounced as the cooling distance diminishes. Recall also that a very reasonable agreement between theory and experiment is observed. The theoretical lines have been obtained by solving Eq.(27) for the parameter values: $J_{c}=3 \times 10^{8} \mathrm{~A} / \mathrm{m}^{2}, \mu_{0} M_{0}=1.17 \mathrm{~T}$. The critical current density of the superconductor was deduced from the data of the manufacturer's data sheet [20].

2) Maximum attainable force.: The values of the levitation force obtained in our experiments are obviously determined by the actual superconducting critical current density of the sample. A couple of questions arise: (i) how far are these values from the maximum forces attainable (Meissner limit) in the specific configuration of our machine? (ii) to what extent should one be concerned about finite size effects when interpreting real data? f.i.: may the superconductor be modeled as a flat infinite medium [3]? We will address them through the numerical model of this paper, together with a dedicated analytical approximation.

The theory developed in this article provides a method to 
evaluate the reference value for any given system. Just, one has to calculate the ZFC behavior for the specific geometry, in the limit $J_{c} \rightarrow \infty$. In practice, this means nothing but minimizing Eq. 27p) and plainly skipping the restriction on $J_{c}$ (just declare $\left|L_{b}\right\rangle$ and $\left|U_{b}\right\rangle$ as empty sets in QP_function). In our geometry, one obtains a levitation force of $405 \mathrm{~N}$ for $d=0$. When the calculation is performed for the actual superconductor in our machine $\left(J_{c}=3 \times 10^{8} \mathrm{~A} / \mathrm{m}^{2}\right)$, the value obtained is $260 \mathrm{~N}$, roughly $64 \%$ of the maximum attainable.

As concerns the second question, an analytical expression is given below that allows to estimate the maximum force between a cylindrical magnet (radius $b$ ) and superconductor (radius $a$ ) in the region $b<a$. Based on [21], when $b / a \rightarrow 0$ one has (infinite superconductor):

$$
\begin{aligned}
F_{\infty} \approx & 2 \mu_{0} M_{0}^{2} b\left[2 \frac{K\left(k_{2}\right)-E\left(k_{2}\right)}{k_{2} / d_{2}}\right. \\
& \left.\left.-\frac{K\left(k_{1}\right)-E\left(k_{1}\right)}{k_{1} / d_{1}}-\frac{K\left(k_{3}\right)-E\left(k_{3}\right)}{k_{3} / d_{3}}\right)\right]
\end{aligned}
$$

where we have defined $k_{n} \equiv b / \sqrt{b^{2}+d_{n}^{2}}$ and $d_{1} \equiv d, d_{2} \equiv$ $d+h_{M} / 2, d_{3} \equiv d+h_{M}$

This expression gives $F_{\infty}=420 \mathrm{~N}$, a value that has to be compared to the result $F=405 \mathrm{~N}$ for our case $(b=0.9 a)$. This implies that the flat superconductor approximation works rather well for obtaining the force at small distances just if one has $b<a$.

\section{CONCLUding REMARKS}

It can be said that the levitation systems based on superconductors and permanent magnets are a promising concept, but their modeling is not a trivial issue. In this work, we provide a set of theoretical tools that allow a precise quantitative analysis of two predominant geometries (rotationally and translationally symmetric) with the least mathematical complication. A unified treatment of both cases is introduced, with inductance matrices $\mathrm{m}$ and a scalar streamfunction, $\psi$ obtained from those, being the central elements to the model. Though grounded on more abstract formalism [12], [13], this application finds a rather tractable layout.

A discretized formulation has been described that easily runs on a personal computer, with a minimal effort, just relying on elementary use of popular computing languages (as OCTAVE or MATLAB ${ }^{\circledR}$ ). The basic procedure may be sketched as follows.

1 Generate the grid points $\left(x_{i}, z_{i}\right)$ that allocate the current circuits equivalent to the components: Eq. 16] with $\lceil i / N\rceil$ just replaced by $\operatorname{ceil}(\mathrm{i} / \mathrm{N})$

2 Apply Eqs. 17, 18, 19p to generate the matrix $\mathrm{m}$.

Next, one introduces the physical process (relative displacements) and iteratively solves the problem, i.e.: gets the supercurrent $g$-vector $\left|\mathrm{J}_{\mathrm{n}}\right\rangle \equiv\left\{\jmath_{i}\left(t_{n}\right)\right\}$ This is done by:

3 Calculate the "updating" vector that gauges the variations of the state variables $\left|\mathrm{A}_{0}\right\rangle=\left|\psi^{P}\right\rangle-\left|\check{\psi}^{P}\right\rangle-\mathrm{m}|\breve{\mathrm{J}}\rangle$.

4 Use built-in solver of the quadratic problem:

$$
|\mathrm{J}\rangle:=\mathrm{QP} \mathrm{P}_{-} \text {function }\left(\mathrm{m},\left|\mathrm{A}_{0}\right\rangle,\left|\mathrm{L}_{b}\right\rangle,\left|\mathrm{U}_{b}\right\rangle, \mathrm{T}\right)
$$

that gives the induced supercurrents, constrained by the bounds $\left|\mathrm{L}_{b}\right\rangle$ and $\left|\mathrm{U}_{b}\right\rangle$, and the transport condition $\mathrm{T}$.

5 Iterate along the desired process by updating $\left|\psi^{P}\right\rangle$.

6 Evaluate forces $\mathbf{f}=P\langle\mathbf{n} \mid \mathrm{J}\rangle$ with the "projector" $\langle\mathbf{n}|$ given by Eq.32

7 Optionally, plot the field lines ("isolines" of $\left.\Psi^{\text {full }}=\Psi^{s c}+\Psi^{P}\right)$, by using the built-in function contour $(\mathrm{x}, \mathrm{z}, \psi)$.

The validation of the theory against experimental data shows that the ansatz of constant material parameters (superconducting critical current density and magnetic remnant field) are justified at $T=77 \mathrm{~K}$ and for the high-quality MT-YBaCuO and $\mathrm{NdFeB}$ materials. Calibration against the ideal maximum forces attainable (Meissner limit) is suggested. For this case, we provide a practical analytical expression (Eq. (37)) that gives a good estimation, just by assuming that the lateral size of the magnet is not bigger than the superconductor's.

Natural extensions of the model that can be implemented with ease would be (i) the consideration of field dependence for the critical current density, (ii) the inclusion of inhomogeneities in the superconductor (as those related to the appearance of granularity [22]) by using a position dependetnt $J_{c}$, (iii) the investigation of the behavior of multicomponent systems, such as levitation tracks with an arbitrary array of magnets, or (iv) the inclusion of paramagnetic materials. A fundamental, but simple modification of the model must be considered in the last instance: additional unknowns related to the effective paramagnetic current densities, as well as a boundary condition related to the material permeability should be incorporated. Assuredly, any of these extensions should keep the $2 \mathrm{D}$ character of the problem.

\section{REFERENCES}

[1] J. R. Hull, "Superconducting bearings", Supercond. Sci. Technol., vol 13, no.2, pp. R1-R15, Feb. 2000 ; K. B. Ma, Y. V. Postrekhin, and W. K. Chu, "Superconductor and magnet levitation devices", Rev. Sci. Instrum., vol. 13, no.2, pp. 4989-5017, Jun. 2003 ; F. Moon, "Superconducting Levitation", Hoboken, NJ: Wiley, 2008.

[2] C. Navau, N. Del-Valle and A. Sánchez, "Macroscopic modeling of magnetization and levitation of hard type-II superconductors: the criticalsate model", IEEE Trans. Appl. Supercond. vol. 23, no. 1, p. 8201023, Feb. 2013

[3] A. A. Kordyuk, "Magnetic levitation for hard superconductors", J. Appl. Phys., vol. 83, no. 1, pp. 610-612, Jan. 1998.

[4] J. R. Hull and A. Cansiz, "Vertical and lateral forces between a permanent magnet and a high-temperature superconductor", J. Appl. Phys., vol. 86, no.11, pp. 6396-6404, Aug. 1999.

[5] C. P. Bean, "Magnetization of high-field superconductors", Rev. Mod. Phys. vol. 36, no. 1, pp. 31-39, Jan. 1964.

[6] X. Zheng and Y. Yang, "Transition Cooling Height of High-Temperature Superconductor Levitation System", IEEE Trans. Appl. Supercond., vol. 17, no. 4, pp. 83-89, Dec. 2007.

[7] D. R. Alonso, T. A. Coombs, and A. M. Campbell, "Numerical solutions to the critical state in a magnet-high temperature superconductor interaction, Supercond. Sci. Technol., vol. 18, no. 2, pp. S209-S214, Feb. 2005.

[8] H. Ueda, S. Azumaya, S. Tsuchiya, and A. Ishiyama, "3D Electromagnetic Analysis of Levitating Transporter Using Bulk Superconductor", IEEE Trans. Appl. Supercond., vol. 16, no. 2, pp. 1092-1095, Jun. 2006

[9] X. Wang, Z. Ren, H. Song, X. Wang, J. Zheng, S. Wang, J. Wang, and Y. Zhao, "Guidance force in an infinitely long superconductor and permanent magnetic guideway system, Supercond. Sci. Technol., vol. 18, no. 2, pp. S99-S104, Feb. 2005. 
[10] G.-T. Ma, J.-S. Wang, and S.-Y. Wang, "3-D Modeling of High-Tc Superconductor for Magnetic Levitation / Suspension Application-

Part I: introduction to the method", IEEE Trans. Appl. Supercond., vol. 20, no. 4, pp. 2219-2227, Aug. 2010; "3-D Modeling of HighTc Superconductor for Magnetic Levitation / Suspension ApplicationPart II: validation with experiment", IEEE Trans. Appl. Supercond., vol. 20, no. 4, pp. 2228-2234, Aug. 2010.

[11] Z. J. Yang, "Levitation force on a permanent magnet over a superconducting plane: modified critical state model", J. Supercond., vol. 10, no. 2, pp. 137-149, April 1997.

[12] A. Badía-Majós and C. López, "Electromagnetics close beyond the critical state: thermodynamic prospect", Supercond. Sci. Technol., vol. 25, no. 10, p. 104004, Oct. 2012.

[13] A. Badía-Majós and C. López, "Modeling current voltage characteristics of practical superconductors", Supercond. Sci. Technol., vol. 28, no. 2, p. 024003 , Feb. 2015.

[14] Though seemingly tautological, the definition of $\Psi_{\mathbf{y}}$ will acquire a clear significance when general 2D problems are treated.

[15] These points are just relevant handicaps in conventional $H$-formulations.

[16] A. Badía-Majós, J. F. Cariñena, and C. López, "Geometric treatment of electromagnetic phenomena in conducting materials: variational principles", J. Phys. A: Math. Gen., vol. 39, no. 47, pp. 14699-14726, Nov. 2006.

[17] F. W. Grover, "Inductance Calculations", Instrument Society of America, Research Triangle Park, NC, 1980.

[18] A. R. Conn, N. I. M. Gould, Ph. L. Toint, "LANCELOT: A Fortran Package for Large-Scale Nonlinear Optimization", Springer Series in Computational Mathematics. Springer Verlag, Berlin, 1998.

[19] C. Navau and A. Sánchez, "Stiffness and energy losses in cylindrically symmetric superconductor levitating systems", Supercond. Sci. Technol. vol. 15, pp. 1445-1453, Sept. 2002.

[20] When the same kind of analysis was applied to the ZFC data provided by the manufacturer (www.can-superconductors.com) an outstanding good agreement between theory and experiment was obtained for $J_{c}=3 \times 10^{8} \mathrm{~A} / \mathrm{m}^{2}$ for a set of 7 different samples.

[21] A. Badía-Majós and H.C. Freyhardt, "Meissner state properties of a superconducting disk in a non-uniform magnetic field", J. Appl. Phys., vol. 83, no. 5. pp. 2681-2688, March 1998.

[22] T. A. Coombs, A. Cansiz, A. M. Campbell, "A superconducting thrustbearing system for an energy storage flywheel", Supercond. Sci. Technol., vol. 15, no. 5. pp. 831-835, May 2002. 